\title{
The Effect of Glucagon-Like Peptide-1 Receptor Agonists on Renal Outcomes in Type 2 Diabetes
}

Win L. Yin · Steve C. Bain · Thinzar Min

Received: January 28, 2020 / Published online: March 17, 2020

(C) The Author(s) 2020

\section{ABSTRACT}

This review examines the available literature on the effect of glucagon-like peptide-1 receptor agonists (GLP-1RAs) on renal outcomes in type 2 diabetes mellitus. Diabetes is an important cause of end-stage renal disease requiring renal replacement therapy, and diabetic kidney disease is an independent risk factor for cardiovascular disease (CVD). GLP-1RAs are proven to be safe in terms of CVD, and some of them have been shown to have a beneficial effect on cardiovascular outcomes. The effect of GLP-1RAs on hard renal endpoints has yet to be established; to date, there have been no published GLP-1RA clinical trials with primary renal endpoints. In this review, we discuss the evidence

Enhanced Digital Features To view enhanced digital features for this article go to: https://doi.org/10.6084/ m9.figshare.11925570.

W. L. Yin · S. C. Bain

Department of Diabetes and Endocrinology,

Singleton Hospital, Swansea Bay University Health

Board, Swansea SA2 8QA, UK

S. C. Bain · T. Min ( $\varangle)$

Diabetes Research Group, Swansea University

Medical School, Swansea SA2 8PP, UK

e-mail: thinzar.min@swansea.ac.uk

\section{T. Min}

Department of Diabetes and Endocrinology, Neath Port Talbot Hospital, Swansea Bay University Health Board, Swansea SA12 7BX, UK for a renal protective role of GLP-1RAs, highlighting the secondary renal outcomes from recent cardiovascular outcome trials of this class of glucose-lowering therapies.

Keywords: Chronic kidney disease; Diabetesrelated nephropathy; Diabetic nephropathy; Dipeptidyl peptidase-4 (DPP-4) inhibitors; Glucagon-like peptide-1 receptor agonists; Type 2 diabetes

\section{Key Summary Points}

Glucagon-like peptide-1 receptor agonists (GLP-1RAs) are safe and effective in patients with renal function decline.

GLP-1RAs reduce the emergence and progression of proteinuria.

The impact of GLP-1RA on estimated glomerular filtration rate (eGFR) decline is marginal.

No benefits have been observed on hard renal endpoints.

Further studies with a unified composite renal endpoint and longer duration are needed. 


\section{INTRODUCTION}

Diabetes is the leading cause of chronic kidney disease (CKD) and $40 \%$ of patients with type 2 diabetes (T2DM) are affected by diabetic kidney disease (DKD) [1]. In addition, DKD is the single most common cause of end-stage renal disease (ESRD) requiring renal replacement therapy (RRT) [2]. Patients with T2DM and DKD are at increased risk of developing cardiovascular disease (CVD), and DKD itself is an independent risk factor for hypertension and premature death. Recent years have seen changes in diabetes management, moving away from a glucocentric approach to multifactorial risk factor modification with a consequent reduction in incidence of T2DM-related CV morbidity and mortality by $>50 \%$. However, the incidence of T2DM-related ESRD decreased by only 29\% [3].

\section{SEARCH STRATEGY}

Publications were identified through searches of Medline, PubMed, Web of Science and Google Scholar. Search terms included 'diabetes kidney disease', 'diabetic nephropathy', 'chronic kidney disease', 'diabetes cardiovascular outcome trials', 'microvascular', 'glucagon-like peptide', 'cardiovascular', 'macrovascular', 'renal outcome' and 'type 2 diabetes'. Articles were excluded if they were case reports, editorials or small studies that the authors felt had methodological limitations. All studies published in English were included and reviewed by the authors. This article is based on previously conducted studies and does not contain any studies with human participants or animals performed by any of the authors.

\section{DIABETIC KIDNEY DISEASE: DIAGNOSIS AND PROGNOSIS}

$\mathrm{DKD}$, also known as diabetic nephropathy, is clinically defined by a persistently elevated urinary albumin: creatinine ratio (UACR) $\geq 30 \mathrm{mg} / \mathrm{g}$ (or $\geq 3.4 \mathrm{mg} / \mathrm{mol}$ ) and/or a sustained reduction in estimated glomerular filtration rate $(\mathrm{eGFR})<60 \mathrm{ml} / \mathrm{min} / 1.73 \mathrm{~m}^{2}$ [4].
Classical pathological changes of DKD are divided into five stages, which occur over a period of 5-15 years. In brief, they are: stage 1 , hyperfunction and hypertrophy of the kidneys; stage 2 , histological morphological changes with increased glomerular filtration rate (GFR) and urinary albumin excretion (UAE) with exercise; stage 3, incipient diabetic nephropathy with mildly abnormal UAE rates termed microalbuminuria; stage 4, overt diabetic nephropathy with UAE $>0.5 \mathrm{~g} / 24 \mathrm{~h}$, hypertension and declining eGFR; stage 5, ESRD [5]. Clinical manifestations of DKD differ between people with type 1 diabetes (T1DM) and those with T2DM. While patients with T1DM typically present with the stages of nephropathy described above, heterogenicity exists in people with T2DM. Thus, patients may present with microalbuminuria and elevated blood pressure, without their being progression through the stages. Patients with T2DM may also have significant co-morbidities such as obesity, hypertension or CVD, which have pre-dated their diagnosis of diabetes.

Microalbuminuria [an albumin excretion rate (AER) of $20-200 \mathrm{mcg} / \mathrm{min}$ ] is the earliest clinical manifestation of DKD in T1DM and thought to be predictive of DKD progression in patients with T2DM. The progression rate from normoalbuminuria to microalbuminuria, and from microalbuminuria to macroalbuminuria, is approximately $2 \% / y e a r$. In the UKPDS study, 15 years after diagnosis, $40 \%$ of patients had macroalbuminuria and $30 \%$ had an eGFR $<60 \mathrm{ml} / \mathrm{min} / 1.73 \mathrm{~m}^{2}$ or a doubling of serum creatinine [6]. The progression of microalbuminuria to macroalbuminuria does not occur in all patients and may regress in some. The progression of albuminuria is influenced by blood pressure, lipids and glycaemic control [7].

\section{GLUCAGON-LIKE PEPTIDE-1 RECEPTOR AGONISTS}

Glucagon-like peptide-1 receptor agonists (GLP1RA) decrease blood glucose levels by stimulating glucose-dependent insulin secretion, inhibiting glucagon secretion, reducing gastric 
emptying, reducing appetite and promoting satiety [8]. Currently, there are six GLP-1RA injectables [exenatide (bid daily), exenatide (once weekly), lixisenatide (once daily), liraglutide (once daily), dulaglutide (once weekly) and semaglutide (once weekly)] approved in Europe and the USA and one GLP1RA oral formulation (semaglutide) in the USA. A further one (albiglutide), has never been launched in the UK and was withdrawn from global use in 2018. Three GLP-1RAs are mainly eliminated by the kidneys and the remaining ones by peptidases and the kidneys. Therefore, all have license limitations based on the stages of CKD (Table 1).

Preclinical studies demonstrated GLP-1 receptor expression in the proximal tubular cells, glomeruli and vascular smooth muscle cells of kidney $[9,10]$. However, the mechanisms for any potential renoprotective effect of GLP-1RA have yet to be established. Proposed mechanisms include renal tubular effects, a renal haemodynamic effect and reduction in renal oxidative stress. The renal tubular effects

Table 1 Glucagon-like peptide-1 receptor agonists and their license limitation as per renal function

\begin{tabular}{ll}
\hline $\begin{array}{l}\text { GLP1 receptor } \\
\text { agonists }\end{array}$ & Renal license limitation \\
\hline Exenatide BD (Byetta) & $\begin{array}{c}\text { eGFR } 30-50 \mathrm{ml} / \mathrm{min} \text { - use with } \\
\text { caution } \\
\text { eGFR }<30 \mathrm{ml} / \mathrm{min} \text {-not } \\
\text { recommended }\end{array}$ \\
$\begin{array}{l}\text { Exenatide QW } \\
\text { (Bydureon) }\end{array}$ & recommended \\
Lixisenatide & eGFR $<30 \mathrm{ml} / \mathrm{min}$-not \\
(Lyxumia) & recommended \\
Liraglutide (Victoza) & eGFR $<15 \mathrm{ml} / \mathrm{min}$ - not \\
& recommended \\
Dulaglutide & eGFR $<15 \mathrm{ml} / \mathrm{min}$ - not \\
(Trulicity) & recommended \\
Semaglutide & eGFR $<15 \mathrm{ml} / \mathrm{min}$ - not \\
(Ozempic) & recommended \\
\hline
\end{tabular}

$e G F R$ estimated glomerular filtration rate of GLP-1 are thought to be due to its natriuretic and diuretic properties. In both animal [11] and human studies involving healthy subjects, as well as subjects with T2DM, GLP-1 infusion was shown to promote natriuresis and diuresis by inhibition of the sodium-hydrogen exchanger 3 (NHE3) localized at the brush border of the renal proximal tubule. Infusion of lixisenatide [12], exenatide [13] and liraglutide [14] in people with T2DM has been shown to reduce urinary sodium reabsorption and increase proximal urinary sodium excretion. Evidence concerning the glomerular haemodynamic effects of GLP-1 are conflicting. Zhou et al. demonstrated that infusion of the GLP-1 RA liraglutide improved sodium and water handling and increased GFR in rats [15]. However, human studies with lixisenatide, exenatide and liraglutide showed no effect on renal haemodynamics (GFR or renal blood flow) [12, 13]. GLP-1 is also thought to have antioxidant properties. GLP-1RA knockout mice showed higher levels of albuminuria and more advanced mesangial expansion. Liraglutide administration in these mice delayed progression of DKD by a reduction in mesangial expansion and reduced levels of glomerular superoxide along with increased levels of renal nitric oxide [16]. Some studies suggested that the renoprotective effect of GLP-1 is mediated by metabolites of innate GLP-1 such as GLP-1 (9-37) and GLP-1 (28-37). In the animal studies, GLP-1 metabolites did not have an effect on glucose metabolism but were associated with lowered expression of renal tubular injury markers and less tubulointerstitial damage by decreasing accumulation of macrophages and $\mathrm{T}$ cells in kidneys [17].

\section{RENAL OUTCOMES IN GLP-1RA CLINICAL TRIALS}

Since the publication of US Food and Drug Administration (FDA) guidance in 2008, robust cardiovascular safety data in patients with T2DM is a prerequisite for approval of glucoselowering therapies [18]. All GLP-1RAs (except Exenatide bid daily) were assessed for cardiovascular safety and some have shown 
Table 2 GLP-RA clinical trials with renal outcome

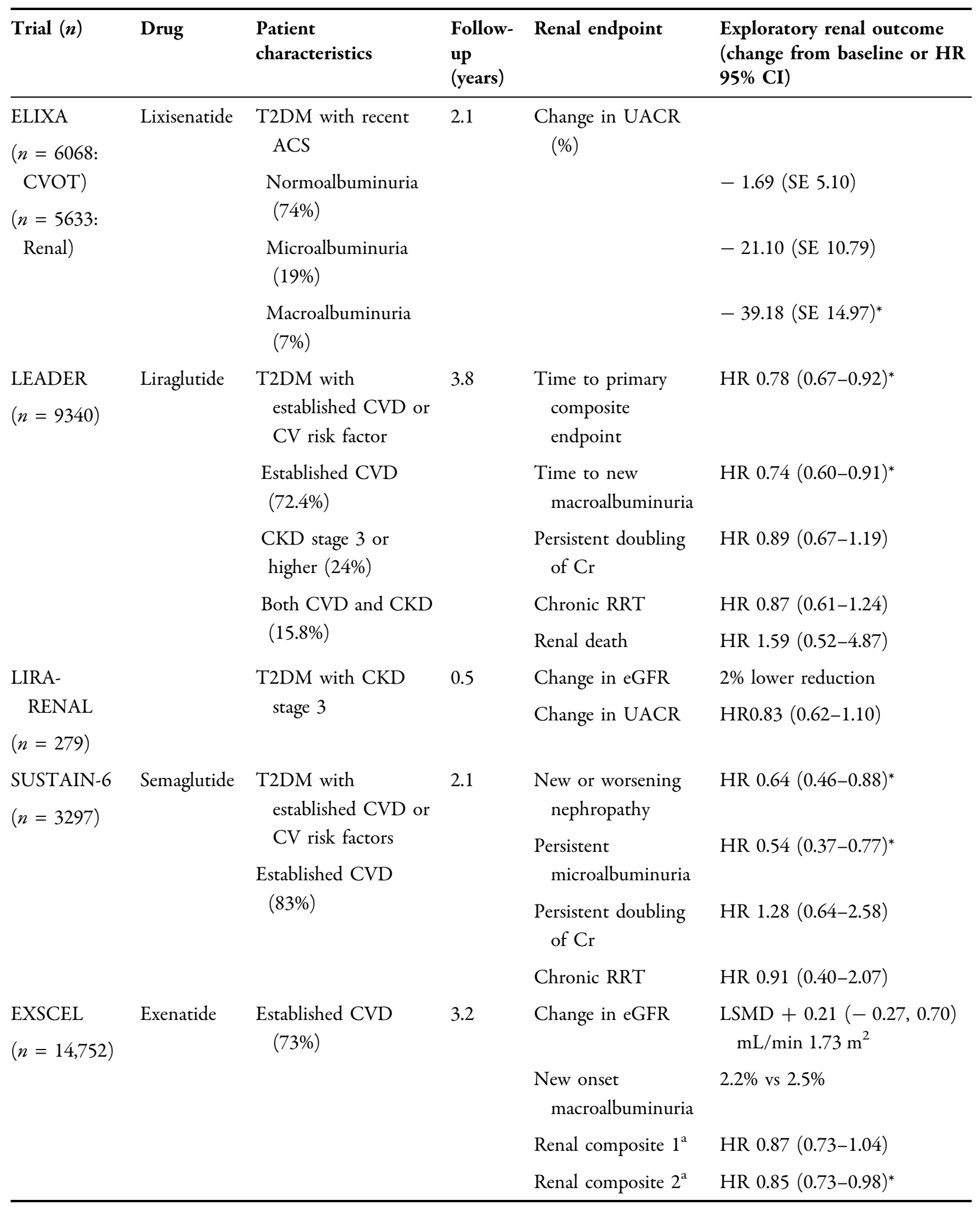


Table 2 continued

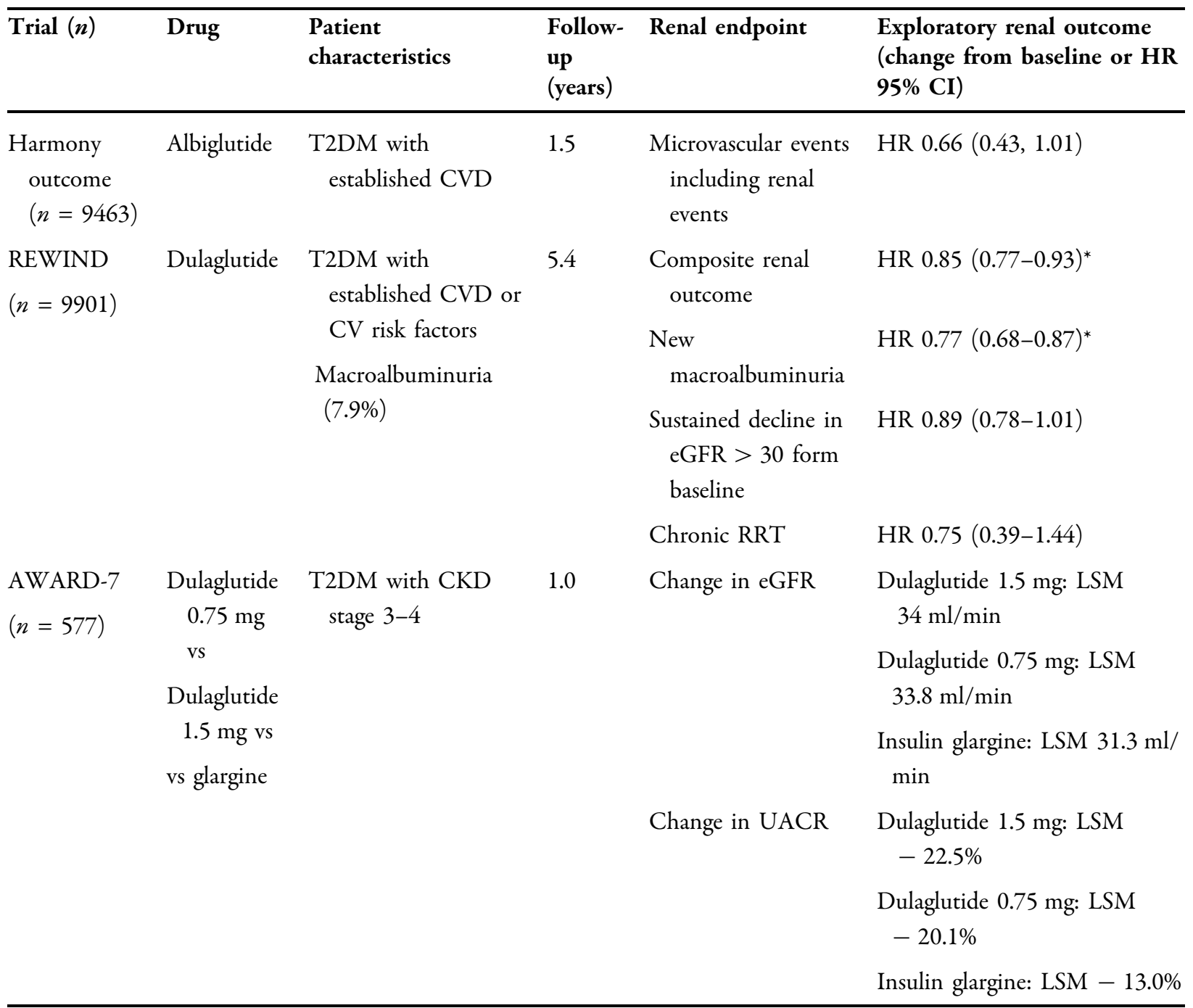

T2DM type 2 diabetes, CVOT cardiovascular outcome trial, $U A C R$ urinary albumin creatinine ratio, CVD cardiovascular disease, $C K D$ chronic kidney disease, $C r$ serum creatinine, $R R T$ renal replacement therapy, eGFR estimated glomerular filtration rate, $H R$ hazard ratio, $C I$ confident interval, $L S M D$ least squared mean difference, $L S M$ least squared mean *Statistically significant

${ }^{a}$ Renal composite 1: 40\% eGFR decline, RRT and renal death; renal composite 2: 40\% eGFR decline, RRT, renal death and new macroalbuminuria

favourable CV outcome, i.e. reduction in major adverse cardiovascular events (MACE). Most of these studies incorporated secondary exploratory renal endpoints; however, to date, there are no published GLP-1RA clinical trials that were designed to assess renal outcomes as a primary endpoint. In this section, we will discuss published secondary renal outcomes of each GLP1RA, in the chronological order of their published cardiovascular outcome trials (CVOTs) (Table 2).

\section{Lixisenatide}

The primary results of the lixisenatide CVOT (ELIXA) was published in 2015, demonstrating non-inferiority of lixisenatide compared with 
placebo in 6068 T2DM patients with a recent acute coronary syndrome [19]. Subsequent renal exploratory analysis, published in 2018, examined the percentage change in UACR and eGFR, according to prespecified albuminuria status at baseline [normoalbuminuria (UACR $<30 \mathrm{mg}$ / $\mathrm{g}$ ); microalbuminuria ( $\geq 30$ to $\leq 300 \mathrm{mg} / \mathrm{g}$ ); macroalbuminuria $(>300 \mathrm{mg} / \mathrm{g}$ )] and time to new-onset macroalbuminuria and doubling of serum creatinine [20]. The UACR data were available for 5978 (99\% of primary ELIXA cohort); 4441 (74\%) had normoalbuminuria, 1148 (19\%) had microalbuminuria, and 389 (7\%) had macroalbuminuria. After a median follow-up of 108 weeks, a significant reduction in UACR [-39.18\%, 95\% confidence interval (CI) -68.53 to $-9.84 ; p=0.007$ ] was observed in patients with macroalbuminuria on lixisenatide compared with those on placebo. The largest eGFR decline from baseline was also observed in the macroalbuminuric group, but no significant differences were observed between the two treatment groups or any UACR subgroups. Lixisenatide was associated with a $20 \%$ relative risk (RR) reduction in new onset macroalbuminuria (HR 0.808; 95\% CI $0.660-0.991 ; p=0.0404)$, when adjusted for baseline haemoglobin A1c (HbA1c), on trial $\mathrm{HbA} 1 \mathrm{c}$ and other renal risk factors. There were no differences in the proportion of patients with adverse renal events [48 (1.6\%) of 3032 patients in the placebo group vs. $48(1.6 \%)$ of 3031 patients in the lixisenatide group] and the proportion of patients with doubling of serum creatinine [35 (1\%) of 3032 patients in the placebo group and 41 (1\%) of 3031 patients in the lixisenatide group (HR 1.163, 95\% CI $0.741-1.825 ; p=0.5127)]$.

\section{Liraglutide}

The LEADER trial assessed the cardiovascular safety of liraglutide $1.8 \mathrm{mg}$ once daily compared with a placebo, in addition to the standard medical care [21]. The trial enrolled 9340 patients with T2DM $(72.4 \%$ had established CVD, 24\% either CKD stage 3 or higher and $15.8 \%$ both CVD and CKD) and the median follow-up was 3.8 years. The LEADER trial reported renal outcomes in its secondary exploratory analysis [22]. The prespecified renal outcome was a composite of new-onset persistent macroalbuminuria, persistent doubling of the serum creatinine, ESRD or death due to renal disease. The trial demonstrated that the liraglutide group had fewer occurrences of the composite renal outcome than the placebo group (268 of 4668 patients vs. 337 of 4672 ; HR $0.78 ; 95 \%$ CI $0.67-0.92 ; p=0.003)$. That was primarily due to a reduction in new-onset persistent macroalbuminuria, which occurred in significantly fewer participants in the liraglutide group than in the placebo group (161 vs. 215 patients; HR 0.74; 95\% CI 0.60-0.91; $p=0.004)$. Numerical reduction in persistent doubling of serum creatinine (87 vs. 97 patients; HR 0.89; $95 \%$ CI $0.67-1.19 ; p=0.43)$ and the need for continuous renal replacement therapy (56 vs. 64 patients; HR 0.87; 95\% CI 0.61-1.24; $p=0.44$ ) were also observed in the liraglutide group compared with the placebo group. There were no significant differences in the rates of renal adverse events between liraglutide and placebo (15.1 events and 16.5 events per 1000 patient-years), including the rate of acute kidney injury (AKI) (7.1 and 6.2 events per 1000 patient-years, respectively).

Another liraglutide study (LIRA-RENAL-a 26-week, randomised controlled double-blind trial, $n=279$ ) was designed to examine the efficacy and safety of liraglutide as an add-on to existing glucose-lowering therapy in people with T2DM and moderate renal impairment (eGFR $30-59 \mathrm{ml} / \mathrm{min} / 1.73 \mathrm{~m}^{2}$ as per MDRD formula) [23]. No significant changes in renal function were observed between the two groups. However, there were a $2 \%$ lower relative difference from baseline eGFR $(p=0.3575)$ and a $17 \%$ RR reduction in UACR (HR $0.83,95 \%$ CI $0.62-1.10 ; p=0.1856$ ) in the liraglutide group compared with placebo.

\section{Semaglutide}

The SUSTAIN-6 trial was designed to assess noninferiority of semaglutide compared with a placebo in terms of cardiovascular safety in patients with T2DM [24]. It enrolled 3297 patients and the median follow-up was 
2.1 years. As was the case in the LEADER trial, it included pre-specified renal microvascular outcomes (defined as persistent macroalbuminuria, doubling of serum creatinine or eGFR $<45$ and RRT). New or worsening nephropathy occurred in 62 patients $(3.8 \%)$ in the semaglutide group and $100(6.1 \%)$ in the placebo group (HR 0.64; $95 \%$ CI $0.46-0.88 ; p=0.005)$. This benefit was once again driven by a fall in new cases of persistent macroalbuminuria $(2.5 \%$ versus $4.9 \%$ of cases) whereas the number of patients who had a doubling of serum creatinine and/or needed continuous RRT was small and similar between groups.

\section{Exenatide}

The EXSCEL trial is the largest CVOT of a GLP1RA, assessing cardiovascular safety of onceweekly exenatide compared with placebo on the background of usual care in 14,752 patients with T2DM (73.1\% had previous CVD) [25]. Renal outcomes in the EXSCEL trial were changes in eGFR, occurrence of new macroalbuminuria and two renal composites (renal composite 1: 40\% decline in eGFR, RRT, renal death and renal composite 2: 40\% decline in eGFR, RRT, renal death and new macroalbuminuria) from a prespecified analysis plan. Intention-to-treat analyses showed no significant difference in eGFR levels and development of new macroalbuminuria but a $15 \%$ lower renal composite 2 adjusted risk with exenatide $(p=0.027)$ [26].

\section{Albiglutide}

The Harmony Outcomes trial was designed to assess cardiovascular safety of once-weekly albiglutide compared with a placebo in patients with T2DM and CVD [27]. The trial included 9463 patients with a median follow-up of 1.5 years. No differences in the rate of eGFR decline and renal events were noted between the two groups.

\section{Dulaglutide}

In the AWARD-7 trial, once-weekly dulaglutide $0.75 \mathrm{mg}$ and $1.5 \mathrm{mg}$ was compared with insulin glargine in 577 patients with T2DM and moderate-to-severe CKD [28]. The secondary outcomes of this trial included renal endpoints: change in eGFR and UACR. At 52 weeks, eGFR decline from baseline was less in both dulaglutide groups $\left(34.0 \mathrm{ml} / \mathrm{min} / 1.73 \mathrm{~m}^{2}\right.$ in dulaglutide $1.5 \mathrm{mg} ; p=0.005 \mathrm{vs}$. insulin glargine and $33.8 \mathrm{ml} / \mathrm{min} / 1.73 \mathrm{~m}^{2}$ in dulaglutide $0.75 \mathrm{mg}$; $p=0.009$ vs. insulin glargine). There was a significant reduction in UACR from baseline in all three treatment groups but no significant differences between treatment groups.

The REWIND study was the cardiovascular safety trial of once-weekly dulaglutide $1.5 \mathrm{mg}$ vs. placebo in 9901 patients with T2DM and a previous cardiovascular event or cardiovascular risk factors [29]. Trial recruits had a baseline mean eGFR of $76.9 \mathrm{ml} / \mathrm{min} / 1.73 \mathrm{~m}^{2}$ and 791 (7.9\%) had macroalbuminuria. The composite renal outcome was defined as the first occurrence of new macroalbuminuria (UACR $>33.9 \mathrm{mg} / \mathrm{mol}$ ), a sustained reduction in eGFR of $30 \%$ or more from baseline or the need for RRT [30]. During a median follow-up of 5.4 years, dulaglutide was associated with a $15 \%$ $\mathrm{RR}$ reduction in the composite renal outcome (HR 0.85, 95\% CI 0.77-0.93; $p=0.0004$ ). Similar to the LEADER and SUSTAIN-6 trials, the renal benefit was driven by the development of new macroalbuminuria, which occurred in 441 (8.9\%) of 4949 patients assigned to dulaglutide and in $561(11.3 \%)$ of 4952 patients on placebo (HR 0.77, 95\% CI 0.68-0.87, $p<0.0001$ ). No significance differences in sustained reduction of eGFR $>30 \%$ and RRT were observed between the two groups. The dulaglutide group had lower eGFR decline [overall LSM difference 0.42 (95\% CI -0.011 to 0.96$) ; p=0.12]$ and lower UACR [overall LSM difference 0.82 (95\% CI $0.78-0.86), p<0.001]$ compared with the placebo group.

\section{RENAL ADVERSE EVENTS}

There have been case reports of AKI [31] and interstitial nephritis associated with GLP-1RA administration [32]. Subsequent cardiovascular outcome studies did not support these findings $[20,22,25,27,30]$ and animal studies have 
reported a renoprotective effect against AKI [33]. One possible explanation is that GLP-1RAinduced AKI might be related to gastrointestinal side effects of GLP-1RA such as severe nausea, vomiting and diarrhoea with subsequent dehydration. The other hypothetical explanation is GLP-1-induced glomerular haemodynamic changes. Gutzwiller et al. suggested a tubuloglomerular feedback mechanism: reduced sodium reabsorption leads to more sodium availability to the macula densa, which in turn activates tubuloglomerular feedback and afferent arteriolar vasoconstriction with a subsequent reduction in eGFR and creatinine clearance [34]. Of note, patients who developed GLP-1-induced AKI were taking stable doses of an angiotensin-converting enzyme inhibitor/ angiotensinogen 2 receptor blocker or diuretics [35].

\section{CONCLUSIONS}

The secondary or exploratory renal outcomes from GLP-1RA cardiovascular outcome trials suggest a renal protective effect of GLP-1RA. A recent meta-analysis of these trials confirms that GLP-1RAs were associated with a $17 \%$ RR reduction in a broad renal composite outcome consisting of occurrence of macroalbuminuria, doubling of serum creatinine or eGFR decline of $\geq 40 \%$, RRT and renal death (HR 0.83; 95\% CI $0.78-0.89 ; p<0.0001$ ) over a median follow-up of 3.2 years [36]. The renal benefit of GLP-1RA was almost entirely driven by reduction in UAE and not by hard renal endpoints such as decline in eGFR, ESRD or kidney-related death. All of the GLP-1RA trials considered above were designed to assess cardiovascular safety and renal outcomes were included as secondary endpoints or exploratory analyses. These studies, therefore, did not have enough power to detect hard-renal outcomes. There is no GLP1RA trial yet published that assesses renal outcomes as a primary endpoint. A placebo-controlled trial of semaglutide by injection with primary renal endpoints (FLOW) [37] is currently on-going and expected to report in 2024. The primary endpoint for the study is time to first occurrence of a composite of persistent
eGFR decline $\geq 50 \%$ from baseline, ESRD (eGFR $<15 \mathrm{ml} / \mathrm{min}$, dialysis or transplantation), death from kidney disease or death from cardiovascular disease. Further long-term trials of GLP-1RAs with primary renal endpoints are needed to firmly establish a clinically relevant renoprotective effect of GLP-1RA rather than reduction in the surrogate marker of UAE. It would also be helpful if such trials could adopt a unified composite renal endpoint [38] (as has been the case with the CVOTs) to allow for meaningful between-trial comparisons and meta-analysis.

\section{ACKNOWLEDGEMENTS}

Funding. No funding or sponsorship was received for this study or the publication of this article.

Authorship. All named authors meet the International Committee of Medical Journal Editors (ICMJE) criteria for authorship for this manuscript, take responsibility for the integrity of the work as a whole, and have given final approval for the version to be published.

Disclosures. Dr. Win L. Yin has nothing to disclose. Prof Steve C. Bain reports grants and personal fees from AstraZeneca, Novo Nordisk, and Sanofi-Aventis; personal fees from Boehringer Ingelheim, Eli Lilly, and Merck Sharp \& Dohme; grants from Medscape; expert advice provided to All-Wales Medicines Strategy Group and National Institute for Health and Care Excellence UK; and partnership in Glycosmedia. Prof Steve C. Bain is also a member of the journal's Editorial Board. Dr. Thinzar Min reports personal fees and travel grants from AstraZeneca, Boehringer Ingelheim, and Napp.

Compliance with Ethics Guidelines. This article is based on previously conducted studies and does not contain any studies with human participants or animals performed by any of the authors. 
Data Availability. Data sharing is not applicable to this article as no datasets were generated or analyzed during the current study.

Open Access. This article is licensed under a Creative Commons Attribution-NonCommercial 4.0 International License, which permits any non-commercial use, sharing, adaptation, distribution and reproduction in any medium or format, as long as you give appropriate credit to the original author(s) and the source, provide a link to the Creative Commons licence, and indicate if changes were made. The images or other third party material in this article are included in the article's Creative Commons licence, unless indicated otherwise in a credit line to the material. If material is not included in the article's Creative Commons licence and your intended use is not permitted by statutory regulation or exceeds the permitted use, you will need to obtain permission directly from the copyright holder. To view a copy of this licence, visit http://creativecommons.org/licenses/by$\mathrm{nc} / 4.0 /$.

\section{REFERENCES}

1. Alicic RZ, Rooney MT, Tuttle KR. Diabetic kidney disease: challenges, progress, and possibilities. Clin J Am Soc Nephrol. 2017;12(12):2032-45.

2. Hole B, et al. UK Renal Registry 19th annual report: chapter 12 multisite dialysis access audit in England, Northern Ireland and Wales in 2015 and 2014 PD one year follow-up: national and centre-specific analyses. Nephron. 2017;137(Suppl 1):269-96.

3. Gregg EW, Williams DE, Geiss L. Changes in diabetes-related complications in the United States. N Engl J Med. 2014;371(3):286-7.

4. Vassalotti JA, Piraino B, Szczech LA. A decade after the KDOQI CKD guidelines: impact on the National Kidney Foundation. Am J Kidney Dis. 2012;60(5): 689-91.

5. Mogensen CE, Christensen CK, Vittinghus E. The stages in diabetic renal disease. With emphasis on the stage of incipient diabetic nephropathy. Diabetes. 1983;32(Suppl 2):64-78.

6. Retnakaran $\mathrm{R}$, et al. Risk factors for renal dysfunction in type 2 diabetes: U.K. prospective diabetes study 74. Diabetes. 2006;55(6):1832-9.
7. Gaede P, et al. Remission to normoalbuminuria during multifactorial treatment preserves kidney function in patients with type 2 diabetes and microalbuminuria. Nephrol Dial Transplant. 2004;19(11):2784-8.

8. Drucker DJ, Nauck MA. The incretin system: glucagon-like peptide-1 receptor agonists and dipeptidyl peptidase- 4 inhibitors in type 2 diabetes. Lancet. 2006;368(9548):1696-705.

9. Pyke C, et al. GLP-1 receptor localization in monkey and human tissue: novel distribution revealed with extensively validated monoclonal antibody. Endocrinology. 2014;155(4):1280-90.

10. Schlatter P, et al. Glucagon-like peptide 1 receptor expression in primary porcine proximal tubular cells. Regul Pept. 2007;141(1-3):120-8.

11. Muskiet MHA, et al. GLP-1 and the kidney: from physiology to pharmacology and outcomes in diabetes. Nat Rev Nephrol. 2017;13(10):605-28.

12. Tonneijck L, et al. Renal tubular effects of prolonged therapy with the GLP-1 receptor agonist lixisenatide in patients with type 2 diabetes mellitus. Am J Physiol Ren Physiol. 2019;316(2):F231-40.

13. Tonneijck L, et al. Acute renal effects of the GLP-1 receptor agonist exenatide in overweight type 2 diabetes patients: a randomised, double-blind, placebo-controlled trial. Diabetologia. 2016;59(7): 1412-21.

14. Skov J, et al. Short-term effects of liraglutide on kidney function and vasoactive hormones in type 2 diabetes: a randomized clinical trial. Diabetes Obes Metab. 2016;18(6):581-9.

15. Zhou X, et al. Acute hemodynamic and renal effects of glucagon-like peptide 1 analog and dipeptidyl peptidase-4 inhibitor in rats. Cardiovasc Diabetol. 2015;14:29.

16. Fujita $\mathrm{H}$, et al. The protective roles of GLP-1R signaling in diabetic nephropathy: possible mechanism and therapeutic potential. Kidney Int. 2014;85(3):579-89.

17. Moellmann J, et al. Glucagon-like peptide 1 and its cleavage products are renoprotective in murine diabetic nephropathy. Diabetes. 2018;67(11):2410-9.

18. FDA. https://www.fda.gov/regulatory-information/ search-fda-guidance-documents/diabetes-mellitusdeveloping-drugs-and-therapeuticbiologics-treat ment-and-prevention. Accessed 3 Oct 2019.

19. Pfeffer MA, et al. Lixisenatide in patients with type 2 diabetes and acute coronary syndrome. N Engl J Med. 2015;373(23):2247-57. 
20. Muskiet MHA, et al. Lixisenatide and renal outcomes in patients with type 2 diabetes and acute coronary syndrome: an exploratory analysis of the ELIXA randomised, placebo-controlled trial. Lancet Diabetes Endocrinol. 2018;6(11):859-69.

21. Marso SP, et al. Liraglutide and cardiovascular outcomes in type 2 diabetes. $\mathrm{N}$ Engl J Med. 2016;375(4):311-22.

22. Mann JFE, et al. Liraglutide and renal outcomes in type 2 diabetes. N Engl J Med. 2017;377(9):839-48.

23. Davies MJ, et al. Efficacy and safety of liraglutide versus placebo as add-on to glucose-lowering therapy in patients with type 2 diabetes and moderate renal impairment (LIRA-RENAL): a randomized clinical trial. Diabetes Care. 2016;39(2):222-30.

24. Marso SP, et al. Semaglutide and cardiovascular outcomes in patients with type 2 diabetes. $\mathrm{N}$ Engl J Med. 2016;375(19):1834-44.

25. Holman RR, et al. Effects of once-weekly exenatide on cardiovascular outcomes in type 2 diabetes. N Engl J Med. 2017;377(13):1228-399.

26. Bethel MA, et al. Microvascular and cardiovascular outcomes according to renal function in patients treated with once-weekly exenatide: insights from the EXSCEL trial. Diabetes Care. 2020;43(2):446-52.

27. Hernandez AF, et al. Albiglutide and cardiovascular outcomes in patients with type 2 diabetes and cardiovascular disease (Harmony Outcomes): a doubleblind, randomised placebo-controlled trial. Lancet. 2018;392(10157):1519-29.

28. Tuttle KR, et al. Dulaglutide versus insulin glargine in patients with type 2 diabetes and moderate-tosevere chronic kidney disease (AWARD-7): a multicentre, open-label, randomised trial. Lancet Diabetes Endocrinol. 2018;6(8):605-17.

29. Gerstein HC, et al. Dulaglutide and cardiovascular outcomes in type 2 diabetes (REWIND): a double- blind, randomised placebo-controlled trial. Lancet. 2019;394(10193):121-30.

30. Gerstein HC, et al. Dulaglutide and renal outcomes in type 2 diabetes: an exploratory analysis of the REWIND randomised, placebo-controlled trial. Lancet. 2019;394(10193):131-8.

31. Dubois-Laforgue D, et al. Severe acute renal failure in patients treated with glucagon-like peptide-1 receptor agonists. Diabetes Res Clin Pract. 2014;103(3):e53-5.

32. Gariani K, de Seigneux S, Moll S. Acute interstitial nephritis after treatment with liraglutide. Am J Kidney Dis. 2014;63(2):347.

33. Chen YT, et al. Exendin- 4 and sitagliptin protect kidney from ischemia-reperfusion injury through suppressing oxidative stress and inflammatory reaction. J Transl Med. 2013;11:270.

34. Gutzwiller JP, et al. Glucagon-like peptide 1 induces natriuresis in healthy subjects and in insulin-resistant obese men. J Clin Endocrinol Metab. 2004;89(6):3055-61.

35. Sharma T, Paixao R, Villabona C. GLP-1 agonist associated acute kidney injury: a case report and review. Diabetes Metab. 2019;45(5):489-91.

36. Kristensen SL, et al. Cardiovascular, mortality, and kidney outcomes with GLP-1 receptor agonists in patients with type 2 diabetes: a systematic review and meta-analysis of cardiovascular outcome trials. Lancet Diabetes Endocrinol. 2019;7(10):776-85.

37. A research study to see how semaglutide works compared to placebo in people with type 2 diabetes and chronic kidney disease (FLOW). 2019. https:// clinicaltrials.gov/ct2/show/NCT03819153. Accessed 23 Oct 2019.

38. Perkovic V, et al. Choice of endpoint in kidney outcome trials: considerations from the EMPA-REG OUTCOME(R) trial. Nephrol Dial Transplant. 2019. https://doi.org/10.1093/ndt/gfz179. 